\title{
QUANTIFYING URBAN VEGETATION COVERAGE CHANGE WITH A LINEAR SPECTRAL MIXING MODEL: A CASE STUDY IN XI'AN, CHINA
}

\begin{abstract}
With the rapid development of urban area of Xi' an in recent years, the contradiction between ecological environmental protection and urban development has become prominent. The traditional remote sensing classification method has been unable to meet the accuracy requirements of urban vegetation monitoring. Therefore, how to quickly and accurately conduct dynamic monitoring of urban vegetation based on the spectral component characteristics of vegetation is urgent. This study used the data of Landsat 5 TM and Landsat 8 OLI in 2011, 2014 and 2017 as main information source and LSMM, region of variation grid analysis and other methods to analyse the law of spatial-temporal change of vegetation components in Xi' an urban area and its influencing factors. The result shows that: (1) The average vegetation coverage of the study area from 2011 to 2017 reached more than $50 \%$, meeting the standard of National Garden City (great than $40 \%$ ). The overall vegetation coverage grade was high, but it had a decreasing trend during this period. (2) The vegetation in urban area of Xi'an experienced a significant change. From 2011 to 2017, only $30 \%$ of the low-covered vegetation, $24.39 \%$ of the medium-covered vegetation and $20.15 \%$ of the high-covered vegetation remained unchanged, while the vegetation in the northwest, northeast, southwest and southeast of the edge of the city's third ring changed significantly. (3) The vegetation quality in urban area of Xi'an has decreased from 2011 to 2014 with $6.9 \%$ of vegetation coverage reduced; while from 2014 to 2017, the overall vegetation quality of this area has improved with $2.1 \%$ of the vegetation coverage increased, which was mainly attributed to urban construction and Urban Green Projects. This study not only can obtain the dynamic change information of urban vegetation quickly, but also can provide suggestions and data support for urban planning of ecological environmental protection.
\end{abstract}

Keywords: urban vegetation changes, vegetation components partition, Linear Spectral Mixing Model (LSMM), vegetation quality, remote sensing

\section{Introduction}

With the formulation and implementation of the national plan for building city clusters and metropolitan areas, China's urbanization rate has reached $59.58 \%$ in 2018 [1]. As more and more rural population is moving to cities, the ecological space in urban areas are threatened and there are prominent contradictions between ecological environmental protection and economic development [2]. In this case, the spatial-temporal change monitoring of urban environment will become more and more important [3]. Urban vegetation is the sum of all kinds of plants such as forests, thickets, hedges, flower beds also situated in green roofs, grasslands, trees and crops owned by parks, campuses, temples, squares, courts, courtyards, streets, farmlands and idle spaces in the city [4-6]. As an important element of urban ecosystem, urban vegetation plays an irreplaceable role

\footnotetext{
${ }^{1}$ College of Landscape Architecture and Art, Northwest A\&F University, Xianyang 712100, China

*Corresponding author: 1jj@ nwafu.edu.cn
} 
in promoting the development of urban production, enriching citizens' life and maintaining ecological equilibrium [7]. For example, it can alleviate "Urban Heat Island", improve regional climate and so on [8].

With the rapid development of urbanization, the change of vegetation coverage has become an important basis of measuring urban ecological status and promoting urban ecological landscape planning $[9,10]$. At present, researches on urban vegetation coverage dynamics that scholars at home and abroad carried out are mainly as follows. (1) Image monitoring based on time series [11]. Du et al. [12] used MODIS NDVI data and meteorological observation data during 2000 to 2016 and adopted maximum synthesis, trend analysis and other related methods to study the distribution characteristics of NDVI in Qinghai-Tibet Plateau at different periods and its relationship with precipitation and temperature. Li et al. [13] studied the law of ecological environment evolution in the Yangtze River Delta based on MODIS influence. But this method generally has a low resolution ratio, so that it is difficult to reveal the subtle change of vegetation. (2) Vegetation change monitoring based on medium-resolution land use classification data. Liu et al. [14] used multi-source remote sensing data to clarify the relationship between land use change and urban ecological environment in urban area of Beijing. Hussein et al. [15] extracted land use classification data by Landsat images and monitored urban vegetation coverage. This method usually depends on land use classification standards, so it is difficult to reflect the dynamic conversion between vegetation in different areas with different coverage grades. (3) Urban land monitoring based on Unmanned Aerial Vehicles (UAV) and high-resolution images [16]. Liang et al. [17] pointed out that the UAV remote sensing in the aspect of urban green space information extraction is very mature, which can effectively evaluate urban green spaces and ecological services. Hashim et al. [18] studied urban green space extraction and vegetation index thresholds using high-resolution images. However, there are some limitations in application and promotion due to high-resolution images' large volume of data and its high cost. Monitoring on urban vegetation change mostly focuses on land use classification, but it is time-consuming and labour-intensive. And the computer classification and recognition algorithms are usually used as statistical regression or training methods, most of which are supervisory modelling analysis [19, 20]. In the current researches based on land use classification, the "majority" method is often used to classify mixed pixels, and vegetation components in non-vegetation pixels are often ignored, resulting in statistical errors. How to eliminate these statistical errors from the perspective of remote sensing mechanism and spectral decomposition and how to find out vegetation components contained in non-vegetation units are worth studying.

$\mathrm{Xi}$ 'an is the only megacity in northwest China and a key city along the Belt and Road Initiative [21]. In 2014, the establishment of Xixian New Area was approved by the State Council; in 2018, the National Development and Reform Commission (NDRC) issued a document to approve the Guanzhong Plain City Cluster Development Plan, clearly proposing to build Xi'an into the ninth National Central City [22]. This development trend indicates that with the urban construction speed of Xi'an, its urban environment is changing. Therefore, it is of great significance to carry out research on vegetation coverage change in Xi'an. This study takes the urban area of Xi'an within the third ring as the study area, using the TM remote sensing data in 2011, 2014 and 2017 as the information source and taking the high spatial resolution Google images of the same period as references to analyse three points as follows. (1) The change of vegetation coverage and vegetation components in Xi'an urban area during the past decade. (2) The law of vegetation spatial 
variation and mutual conversion in urban area of Xi'an. (3) The influence factors of vegetation change in urban area of $\mathrm{Xi}$ 'an. The results of this research will provide references for urban vegetation construction and green space system planning in the study area.

\section{Materials and methods}

\section{Study area}

$\mathrm{Xi}$ 'an, the study area, is located between $107^{\circ} 40^{\prime} \sim 109^{\circ} 49^{\prime} \mathrm{E}$ and $33^{\circ} 39^{\prime} \sim 34^{\circ} 45^{\prime} \mathrm{N}$ (Fig. 1). The south and southeast of this city is bounded by the main ridge of the Qinling Mountains, which is adjacent to Hanzhong City and Shangluo City; the west of this city is bounded by Taibai Mountain and Qinghua Taiyuan, bordering Baoji City; the northwest is bounded by the Weihe River, facing Xianyang City across the river; and the east is bounded by the Linghe River and Ruyuan Mountains, bordering Weinan City. The climate of this area belongs to the Semi-humid Monsoon Climate of Warm Temperature Zone with four distinct seasons.

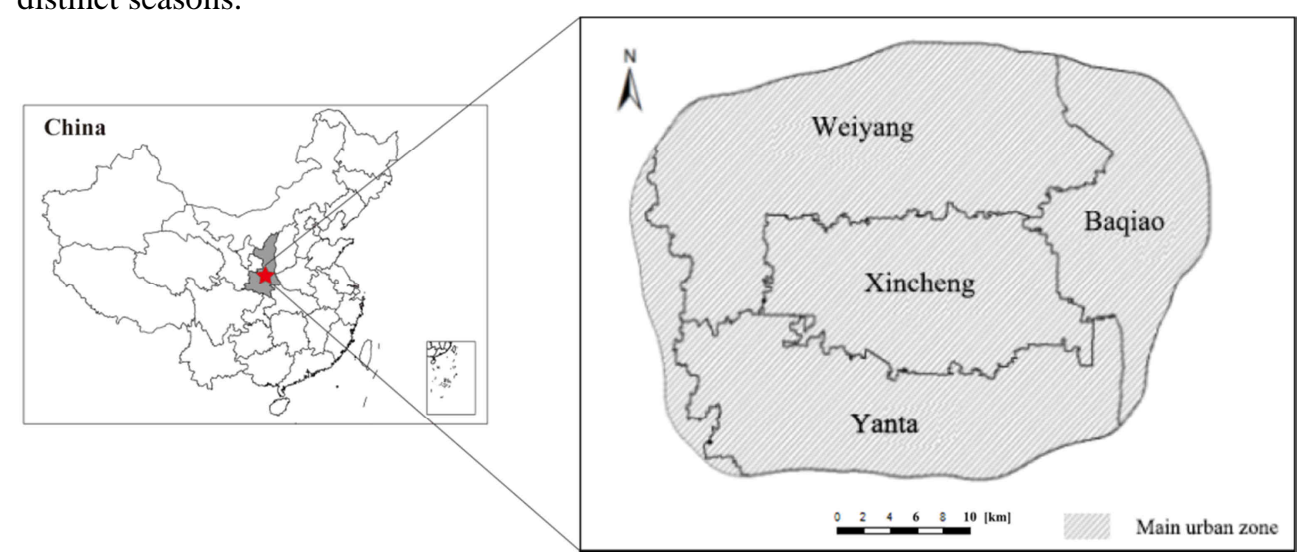

Fig. 1. Study area

\section{Data source and pre-processing}

The remote sensing data in this study comes from the Geospatial Data Cloud of Chinese Academy of Sciences and the administrative boundary data of the study area comes from Xi'an Database of Forest Resources Second Inventory. Considering that the cloud cover on the day when the remote sensing data was obtained was less than $10 \%$ and the influences of vegetation growth season and other factors, 6 bands of the Landsat 5 TM sensor on June 4, 2011 and 7 bands of Landsat 8 OLI sensor on May 11, 2014 and April 17, 2017 were finally selected, with a spatial resolution of $30 \mathrm{~m}$. The orbit number is 127/36. For eliminating the effects of different data source and time, we used relative radiometric calibration method, while treated the Landsat 5 TM images as reference and other images conduct relative calibration. All the calibrations including FLAASH atmospheric correction and geometric correction for the original images was conducted with ENVI 5.3 [23] software. 


\section{Extraction of vegetation coverage grade}

Remote sensing inversion method establishes correlation with vegetation coverage grade through establishing vegetation remote sensing spectral information of the study area, and then obtains vegetation coverage grade [24-26]. When the interaction between incident radiation and the land-cover material in the scene involves a single endmember, then the pixel's spectral response can be satisfactorily modelled by the weighted sum of the endmembers' spectral responses, with the weights estimated by the fraction of the pixel covered by the endmember. The modelling process is then known as Linear Spectral Mixture Model (LSMM) [27]. The Linear Spectral Mixing Model (LSMM) decomposition adopted in this study does not require the measurement of ground vegetation coverage data for modelling which is easy to popularize [28]. Generally, LSMM's definition is that the reflectance of pixels in a certain spectral band is a linear combination of the reflectance of the terminal units constituting pixels and its proportion in the pixel area as the weight coefficient [29-31]. The formula is as follows:

$$
\begin{aligned}
R_{i \lambda}= & \sum_{k=1}^{N} f_{k i} C_{k \lambda}+\varepsilon_{i \lambda} \\
& \sum_{k=1}^{N} f_{k i}=1 \\
R M S= & {\left[\sum_{i=1}^{\lambda}\left(\varepsilon_{i \lambda}\right)^{2} / N\right]^{1 / 2} }
\end{aligned}
$$

where $R_{i \lambda}$ is the spectral reflection of the $i$ pixel in the $\lambda$ band; $N$ is the number of basic components; in $N \leq m+1, m$ is the number of bands; $f$ is the value of component occupied by the $k$ basic component in its corresponding $i$ pixel; $C_{k \lambda}$ is the spectral reflection of the $k$ basic component in the $\lambda$ band; $\varepsilon_{i \lambda}$ is the error value of the $i$ pixel in the $\lambda$ band; RMS is the error image of root-mean-square.

\section{Endmembers collection}

According to the characteristics of land cover in this study area, the endmembers can be defined as four categories: bare soil, vegetation, impervious layer and water body. Generally, there are many methods to collect endmembers, such as endmembers extraction based on geometric vertex, Pixel Purity Index (PPI) or Sequential Maximum Angle Convex Cone (SMACC), etc. [32] In this study, the endmembers were selected by establishing Region of Interest (ROI). Generally, ROI is defined as selecting and applying information about the area you want to capture in remote sensing image processing. First, it used Minimum Noise Fraction (MNF) transformation to remove the noise of mask data and confirm the intrinsic dimensions of data, so as to reduce the amount of calculation in subsequent study [33-35]. In this way, the images in three stages were changed into six band components through MNF transformation. This study synthesized the bands of the first three components to obtain false colour images. Then, based on the Google Earth historical images of the same period as a reference, ROI was established. At last, the original range of the endmembers that influence the spectrum was collected [36] (Fig. 2). 

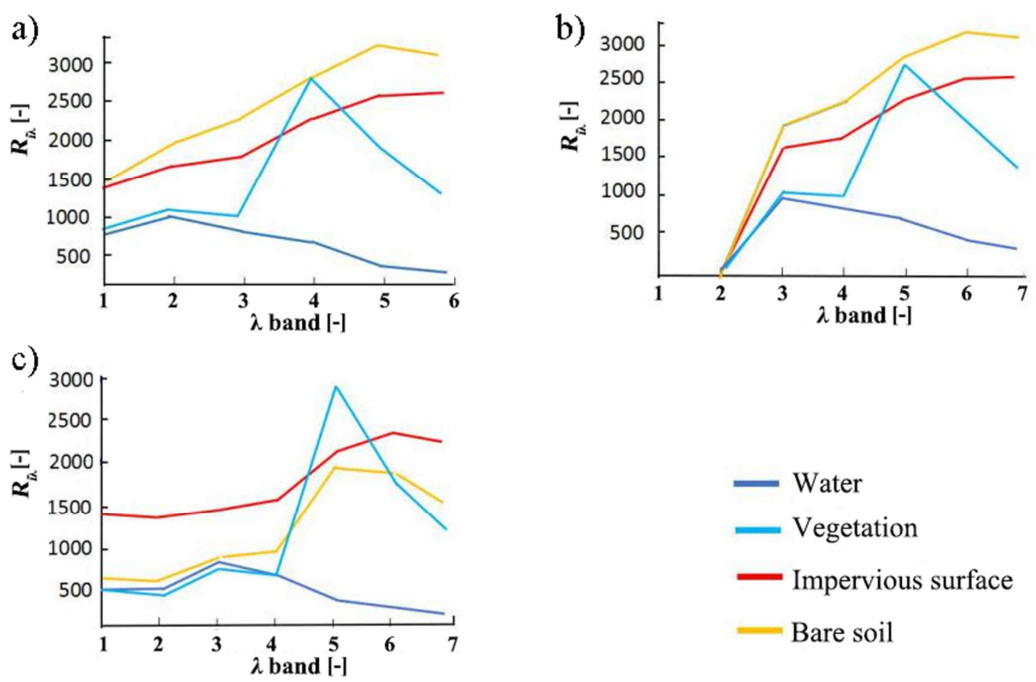

Fig. 2. Spectral reflectance of each band: a) in 2011, b) in 2014, c) in 2017

\section{LSMM decomposition and validation}

Using the collected spectral profile of endmembers, the vegetation coverage of this study area was obtained through LSMM decomposition with the restrictive Least Squares. According to the RMS images of data's root-mean-square in three stages obtained through LSMM decomposition, the mean error was 1.69 , which met the analysis accuracy requirements of vegetation coverage change.

\section{Changes of vegetation coverage grade}

Vegetation components extracted through LSMM were defined as vegetation coverage grades. According to the realities of the study area, the vegetation coverage in this area was divided into five grades (Table 1) using the classification method proposed in the literature [37] and was denoted by $F_{g}$. Then the spatial distribution of vegetation coverage grades is obtained. Through transition matrix and hierarchical encoding (Table 1), changing trends of vegetation coverage grades from 2011 to 2014 and from 2014 to 2019 are calculated. The formula is as follows [38]:

$$
Y_{i \times j}=X_{t i \times j} \times 10+X_{(t+1) i \times j}
$$

where $i, j$ represent the line and column code number of raster graphics; $Y_{i \times j}$ represents vegetation coverage change of pixel in the line $i$, column $j$ from $t$ to $t+1$ period; $X_{k i \times j}$ represents the code number of the previous vegetation coverage; and $X_{(k+1) i \times j}$ represents the code number of the latter vegetation coverage [39].

Table 1

Thresholds of different vegetation coverage grades $\left(F_{g}\right.$ : fraction of vegetation coverage)

\begin{tabular}{|c|c|c|c|c|c|}
\hline Vegetation coverage & No-covered & Low-covered & Medium-covered & High-covered & Full-covered \\
\hline Grade & 1 & 2 & 3 & 4 & 5 \\
\hline Coverage degree [\%] & $0 \leq F_{g}<20$ & $20 \leq F_{g}<40$ & $40 \leq F_{g}<60$ & $60 \leq F_{g}<80$ & $80 \leq F_{g}$ \\
\hline
\end{tabular}




\section{Analysis of vegetation quality grade change based on geospatial grid method}

This study used grid analysis to describe the spatial distribution of vegetation change [40]. Considering the area and the reasonable form of the vegetation distribution of the study area comprehensively, $480 \times 480 \mathrm{~m}$ grids were divided. The attributes of vegetation evolution type in the grid are defined according to the weight of the vegetation change type in the grid. If the weight of vegetation evolution type in grid is more than $50 \%$, then define this type as grid transition. In this way, the main spatial distribution of vegetation evolution types can be obtained.

The vegetation change types were divided based on the transition matrix and the spatial distribution of vegetation coverage grade. The increase of vegetation coverage grade is defined as high-transition type, an unchanged vegetation coverage grade is defined as invariant-transition type, and a decline in vegetation coverage grade is defined as low-transition type. The formula is presented as follows:

$$
T Y_{i \times j}=X_{(t+1) i \times j}-X_{t i \times j}
$$

where $T Y_{i \times j}$ represents vegetation coverage change type from $t$ to $t+1$ period; $X_{(t+1) \times j}$ represents the code number of the previous vegetation coverage; $X_{k i \times j}$ represents the code number of the latter vegetation coverage (Table 1 ). If $T Y_{i \times j}>0$, the vegetation type is defined as high-transition type; if $T Y_{i \times j}<0$, the vegetation type is defined as low-transition type; and if $T Y_{i \times j}=0$, it is defined as invariant-transition type.

\section{Results and discussion}

\section{Vegetation components characteristics in Xi'an urban area}

The average vegetation coverage of the study area in three stages reached $50 \%$, and the coverage rate was relatively high (Fig. 3, Table 2), which exceeded the evaluation standard of National Forest City (great than $40 \%$ ). The annual average vegetation coverage decreased by $11 \%$ from 2011 to $2014,3 \%$ from 2014 to 2017 , and $13 \%$ from 2011 to 2017 respectively. The average reduction of standard deviation of vegetation coverage grade in study area during these three stages was $1.73,6.17,7.90 \%$ respectively, where mainly occurred in the surrounding of the urban city. From the area of different vegetation coverage grades, it can be seen that there is an increasing trend of no vegetation coverage during all these three stages where mainly occurred in the north and northwest of urban area; in the variation trend of low, medium, high, and full vegetation coverage, except that low coverage in 2011 to 2014 and full coverage in 2014 to 2017 increased slightly, the rest of them showed different degrees of downward trend.

Table 2

Statistics of average vegetation coverage and its grade composition area between three stages of the study area

\begin{tabular}{|c|c|c|c|c|c|c|}
\hline \multirow{2}{*}{ Year } & \multicolumn{6}{|c|}{ Area of different vegetation coverage $\left[\mathrm{km}^{2}\right]$} \\
\hline & $\boldsymbol{F}_{g}$ & No coverage & Low coverage & Medium coverage & High coverage & Full coverage \\
\hline 2011 & 58.00 & 190.28 & 94.72 & 75.33 & 50.72 & 46.70 \\
\hline 2014 & 48.00 & 238.58 & 99.20 & 58.24 & 32.87 & 28.86 \\
\hline 2017 & 45.00 & 251.80 & 76.79 & 51.27 & 32.72 & 45.15 \\
\hline$\Delta 14-11$ & -11.00 & 48.30 & 4.47 & -17.09 & -17.85 & -17.84 \\
\hline$\Delta 17-14$ & -3.00 & 13.22 & -22.40 & -6.97 & -0.14 & 16.30 \\
\hline$\Delta 17-11$ & -13.00 & 61.52 & -17.93 & -24.06 & -17.99 & -1.54 \\
\hline
\end{tabular}


a)

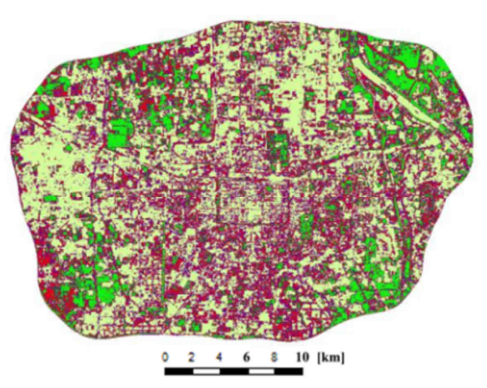

c)

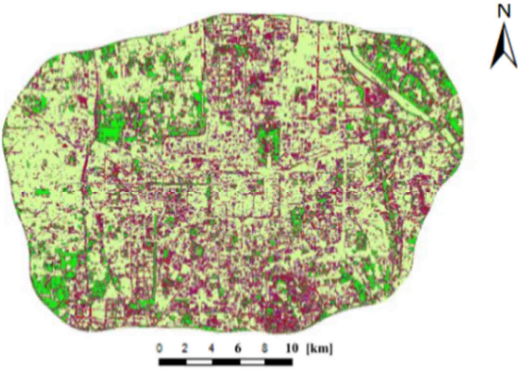

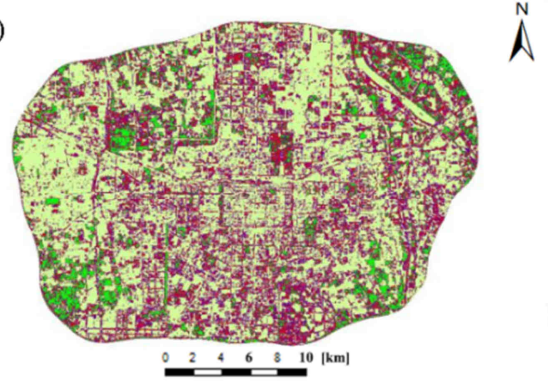

$\stackrel{N}{N}$
$\bigwedge^{N} \quad$ b)

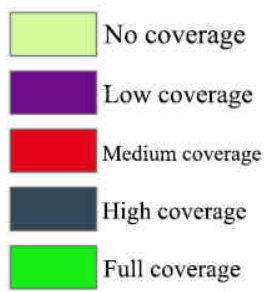

Fig. 3. Vegetation coverage grade: a) in 2011, b) in 2014, c) in 2017

\section{Transitions of vegetation coverage grades in urban area of Xi'an}

During 2011 to 2017, areas without vegetation coverage were located in impervious areas (located in the core of urban city) such as urban buildings, water bodies, etc., among which $83.12 \%$ remained unchanged (Table 5, Fig. $4 \mathrm{c}$ ). The rest was mainly transferred to low and medium coverage grades. The transition to low coverage areas were located at the north, south and southwest to central part of study area; and the transition to medium coverage areas were located at the north and south of study area. Areas that transferred to no coverage grade include all kinds of coverage grade components. The comprehensive transition effect led to a $7.90 \mathrm{~km}^{2}$ reduction of no coverage area and the proportion of low coverage area remained unchanged. The proportion of the rest areas that mainly transferred to no coverage, medium coverage, high coverage and full coverage accounted for 50.10, $13.28,3.91$ and $2.71 \%$ respectively.

Table 3

The transition matrix of different vegetation coverage grade area from 2011 to 2014

\begin{tabular}{|c|c|c|c|c|c|c|}
\hline \multirow{3}{*}{ Transition rate [\%] } & \multicolumn{6}{|c|}{$\mathbf{2 0 1 4}$} \\
\cline { 2 - 7 } & Grade & No coverage & Low coverage & $\begin{array}{c}\text { Medium } \\
\text { coverage }\end{array}$ & High coverage & Full coverage \\
\hline \multirow{3}{*}{2011} & No coverage & 86.93 & 10.22 & 2.04 & 0.58 & 0.24 \\
\cline { 2 - 7 } & Low coverage & 44.61 & 42.97 & 9.41 & 1.98 & 1.02 \\
\cline { 2 - 7 } & $\begin{array}{c}\text { Medium } \\
\text { coverage }\end{array}$ & 20.37 & 35.70 & 31.06 & 8.68 & 4.19 \\
\cline { 2 - 7 } & High coverage & 16.76 & 15.55 & 31.58 & 24.99 & 11.12 \\
\cline { 2 - 7 } & Full coverage & 15.73 & 8.63 & 12.82 & 22.87 & 39.96 \\
\hline
\end{tabular}



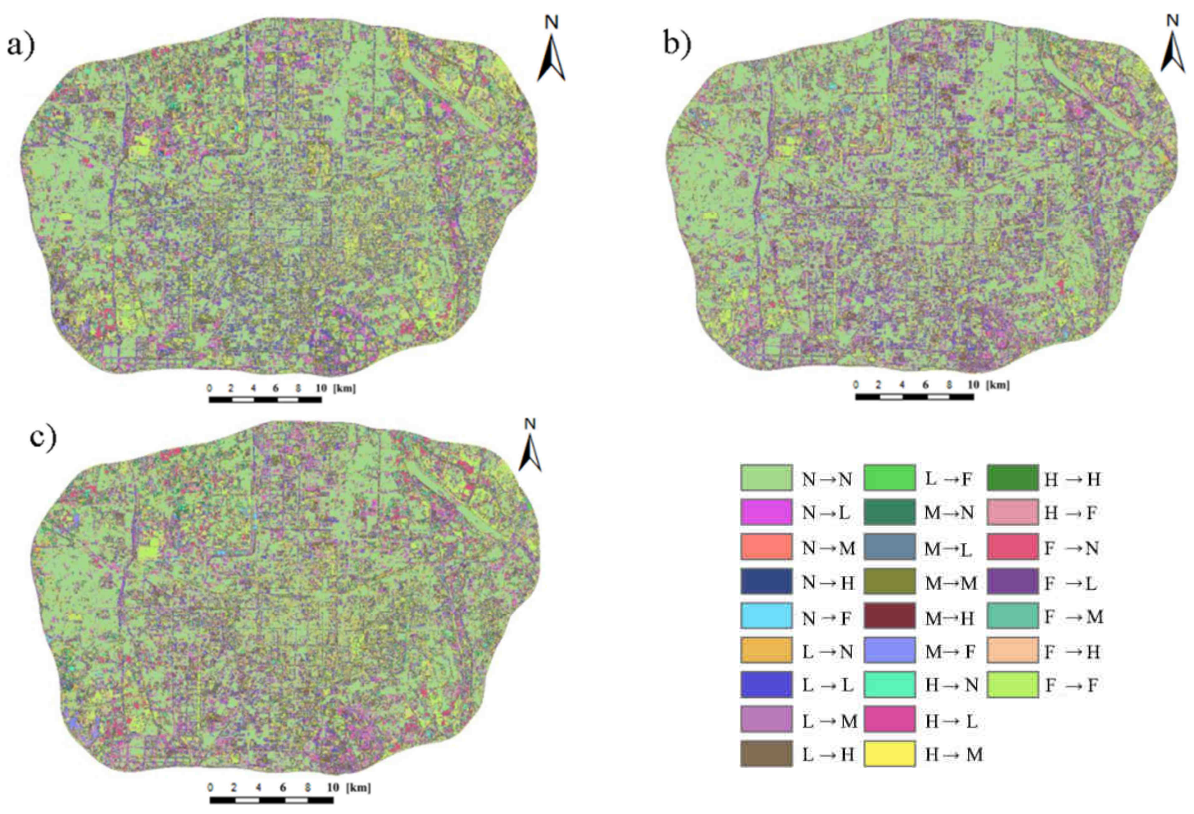

Fig. 4. a) Transfer distribution maps of area proportion of vegetation coverage grade from 2011 to 2014, b) transfer distribution maps of area proportion of vegetation coverage grade from 2014 to 2017, c) transfer distribution maps of area proportion of vegetation coverage grade from 2011 to 2017, note: $\mathrm{N}$ means no coverage, $\mathrm{L}$ means low coverage, $\mathrm{M}$ means medium coverage, $\mathrm{H}$ means high coverage, $\mathrm{F}$ means full coverage

The transition matrix of different vegetation coverage grade area from 2014 to 2017

\begin{tabular}{|c|c|c|c|c|c|c|}
\hline \multirow{3}{*}{ Transition rate [\%] } & \multicolumn{7}{|c|}{$\mathbf{2 0 1 7}$} \\
\cline { 2 - 7 } & Grade & No coverage & Low coverage & $\begin{array}{c}\text { Medium } \\
\text { coverage }\end{array}$ & High coverage & Full coverage \\
\hline \multirow{3}{*}{2014} & No coverage & 84.80 & 11.11 & 2.56 & 0.84 & 0.68 \\
\cline { 2 - 7 } & Low coverage & 33.16 & 36.66 & 21.98 & 5.69 & 2.51 \\
\cline { 2 - 7 } & $\begin{array}{c}\text { Medium } \\
\text { coverage }\end{array}$ & 15.59 & 17.05 & 29.35 & 24.04 & 13.97 \\
\cline { 2 - 8 } & High coverage & 12.79 & 7.17 & 13.36 & 23.81 & 42.87 \\
\cline { 2 - 8 } & Full coverage & 12.65 & 4.65 & 6.18 & 11.25 & 65.28 \\
\hline
\end{tabular}

The transition matrix of different vegetation coverage grade area from 2011 to 2017

Table 5

\begin{tabular}{|c|c|c|c|c|c|c|}
\hline \multirow{3}{*}{ Transition rate [\%] } & \multicolumn{7}{|c|}{$\mathbf{2 0 1 7}$} \\
\cline { 2 - 7 } & Grade & No coverage & Low coverage & $\begin{array}{c}\text { Medium } \\
\text { coverage }\end{array}$ & High coverage & Full coverage \\
\hline \multirow{3}{*}{2011} & No coverage & 83.12 & 10.66 & 3.73 & 1.47 & 1.02 \\
\cline { 2 - 7 } & Low coverage & 50.10 & 30.00 & 13.28 & 3.91 & 2.71 \\
\cline { 2 - 7 } & $\begin{array}{c}\text { Medium } \\
\text { coverage }\end{array}$ & 29.98 & 22.71 & 24.39 & 13.25 & 9.67 \\
\cline { 2 - 8 } & High coverage & 25.14 & 12.77 & 17.22 & 20.15 & 24.72 \\
\cline { 2 - 8 } & Full coverage & 24.07 & 9.01 & 9.35 & 12.89 & 44.69 \\
\hline
\end{tabular}


During 2011 to 2014, areas without vegetation coverage were widely located in impervious areas (located in the core of urban city) such as urban buildings, water bodies, etc. (Table 3, Fig. 4a), among which $86.93 \%$ remained unchanged. The other $13.07 \%$ mainly transferred to low and medium coverage grades which were mainly located at the central of Weiyang District, the south of Baqiao District and Yanta District. Areas that transferred to no coverage grade included all kinds of coverage grade components. The comprehensive transition effect led to a $48.30 \mathrm{~km}^{2}$ increase of no coverage area and the proportion of low coverage area remained unchanged (42.97\%). The proportion of the rest areas that mainly transferred to no coverage and medium coverage accounted for $44.61 \%$ and $9.41 \%$ respectively; areas transferred to no coverage were distributed all over the study area; areas that transferred to no coverage, medium coverage, high coverage and full coverage had a comprehensive impact that resulted in the increase of the low coverage area by $4.47 \mathrm{~km}^{2}$.

During 2014 to 2017, areas without vegetation coverage were widely located in impervious areas such as urban buildings, water bodies, etc., among which $84.80 \%$ remained unchanged. The rest $15.20 \%$ mainly transferred to low and medium coverage grades which were mainly located at the north, southwest, southeast and east of the study area (Table 4, Fig. 4b). Areas that transferred to no coverage grade included all kinds of coverage grade components. The comprehensive transition effect led to a $13.22 \mathrm{~km}^{2}$ increase of no coverage area and the proportion of low coverage area remained unchanged $(36.66 \%)$. The proportion of the rest areas that mainly transferred to no coverage, medium coverage, high coverage and full coverage accounted for 33.16, 21.98, 5.69, $2.51 \%$ respectively; areas transferred to no coverage and medium coverage were distributed all over the study area and areas transferred to high coverage and full coverage were mainly distributed at the fringe of study area; areas that transferred to no coverage, medium coverage, high coverage and full coverage had a comprehensive impact that resulted in the decrease of the low coverage area by $22.40 \mathrm{~km}^{2}$.

\section{Spatial dynamics of quality grade of vegetation coverage}

There were differences in vegetation coverage grade changes among 2011, 2014 and 2017. The rise of vegetation coverage grade is considered to have a trend of vegetation improvement; the invariable vegetation coverage grade is considered to have an unchanged trend; and the decline of vegetation coverage grade is considered to have a trend of vegetation degradation [41-43]. From 2011 to 2014 (Fig. 5a), $6.9 \%$ of vegetation coverage reduced in the study area. The dynamic changes of vegetation coverage in study area were mainly located at the west of Weiyang District, the east of Xincheng District, the north of Baqiao District and the west of Yanta District. The number of grids with declining vegetation coverage grade was greater than that of grids with increasing vegetation coverage grade, indicating that these areas had a trend of vegetation degradation (Fig. 5). From 2014 to 2017 (Fig. 5b), $2.1 \%$ of the vegetation coverage increased in the study area. The dynamic changes of vegetation coverage in study area were mainly located at the west of Weiyang District, the north of Baqiao District and the southeast of Yanta District. The number of grids with increasing vegetation coverage grade was greater than that of grids with declining vegetation coverage grade, indicating that these areas had a trend of vegetation improvement. From 2011 to 2017 (Fig. 5c), the location of dynamic change of vegetation coverage in study area was similar to that from 2014 to 2017 . The number of grids with increasing vegetation coverage grade was slightly less than that of grids with 
declining vegetation coverage grade and the grids density was relatively large, indicating that the overall trend of vegetation improvement was similar to that of vegetation degradation during the past six years.
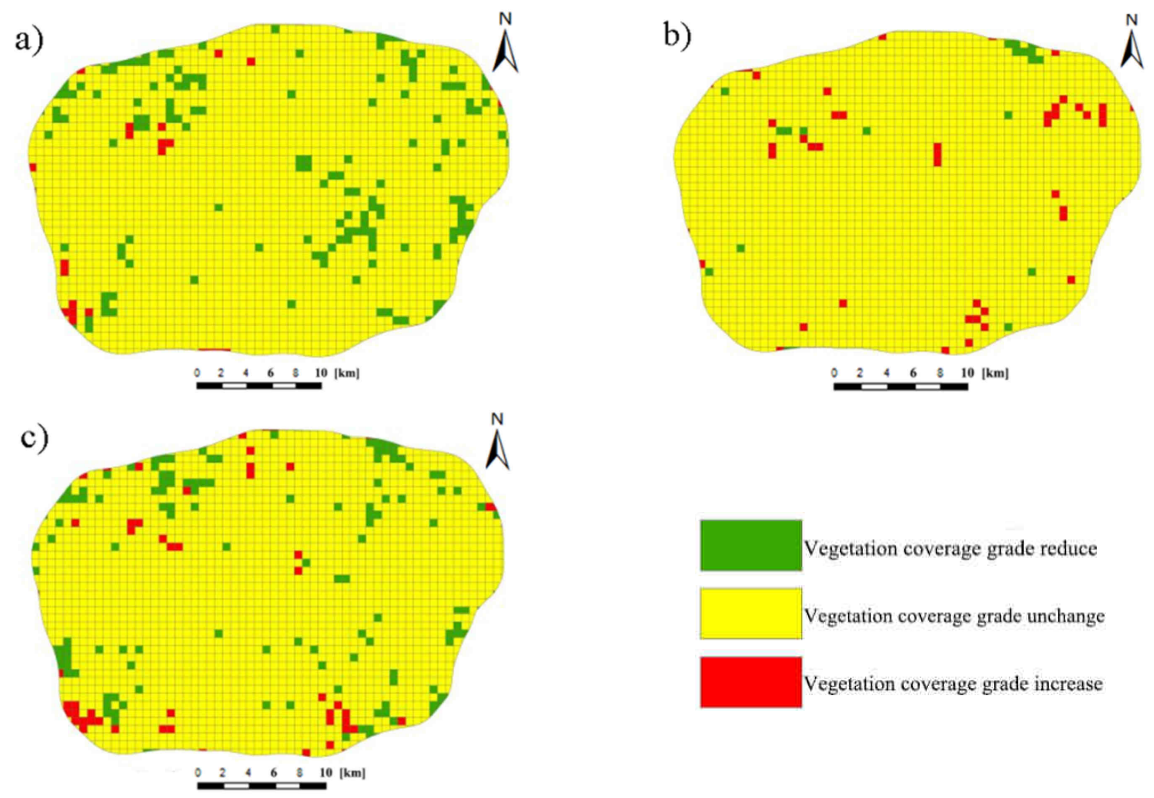

Fig. 5. Distribution of vegetation coverage grade dynamic: a) from 2011 to 2014, b) from 2014 to 2017, c) from 2011 to 2017

Through the results analysis of this study, it can be seen that the vegetation coverage within the third ring of the main urban area of Xi' an has undergone different degrees of quantitative changes and spatial changes in the past six years. This change was in line with the law of general urban development. Generally, the changes in city's central area were relatively small, while the changes in the urban fringe were relatively large, which was the results that influenced by combined factors such as nature, man-made factors, urban socio-economic development and so on. But it was mainly affected by the following factors:

(1) Urban infrastructure had an impact on vegetation coverage change. With the development of this study area and the introduction of new urban planning, the urban construction of Xi'an was strengthened constantly. According to the survey data of Xi'an Bureau of Planning and Natural Resources [44], during the three stages from 2011 to 2017, Xi'an completed a batch of comprehensive urban plans, such as Research on the overall Planning and Development Strategy of Xi'an International Metropolis, Overall Urban Planning of Greater Xi'an and so on. These construction measures affected the change of land using types for urban construction and thus indirectly affected the change of vegetation coverage. According to the Xi'an Statistical Yearbooks, the areas of urban construction land using and road areas in 2011,2014 , and 2017 increased by 36.45 and $24.26 \%$ on average. With the demand of 
urban construction, some areas that used to be low and medium vegetation coverage transferred to construction land, resulting in a reducing trend of vegetation coverage in a short-term. However, this trend will be improved and enhanced with the construction of the Urban Green Space System.

(2) Landscape engineering had an impact on vegetation coverage change. There were 92 newly built or renovated green space squares in Xi'an in 2011, 60 in 2014, and 62 in 2017 [43]. At the same time, the afforestation area in the study area was $6.66 \mathrm{~km}^{2}$ in $2011,7.06 \mathrm{~km}^{2}$ in $2014,1.24 \mathrm{~km}^{2}$ in 2017 . We can see that the increase of green square areas and afforestation areas obviously affected the improvement of vegetation coverage grade and effectively promotes the change of it.

(3) To some extent, the improvement of vegetation coverage was affected by the climate change, because of air temperate and precipitation increased in recent decades, beside the effects of urban heat island could not be neglected. As it is well known, the decision of government had the largest effects on the coverage changes, because the effects of tree species and tree ages selection were greater than the growth of trees. Therefore, selecting the trees that could adapt to local soil and climate conditions is crucial for sustaining a health urban ecosystem.

\section{Conclusion}

Research on the change of urban vegetation coverage plays a significant role in formulating and adjusting the urban vegetation construction planning and improving he urban human settlement environment. Meanwhile, it can provide a basis for the fine management of urban vegetation. The conclusions of this study are as follows:

(1) During 2011 to 2017 , the vegetation construction within the third ring of the main urban area of Xi'an has achieved remarkable results. The results of the study showed that although it fluctuated in different periods, the vegetation coverage declined in 2011 compared with that in 2014 or 2017 , with an average decline of $12 \%$. However, the average vegetation coverage in these six years was above $50 \%$, which exceeded the evaluation standard $(40 \%)$ of National Forest City. The fluctuation of vegetation coverage affected by the development of urban infrastructure in a short-term is a normal phenomenon which does not affect the overall increase trend of urban vegetation coverage.

(2) The spatial distribution of vegetation coverage in the main urban area of Xi'an is a dynamic process. In addition to the relatively stable changes in the central area, the northwest, northeast, southwest, and southeast fringe areas of study area had different degrees of change, which was consistent with the urban development progress of Xi'an in the past six years.

(3) The geospatial grid analysis method of urban vegetation changes based on LSMM can intuitively express changes of vegetation quality. From 2011 to 2014, three stages vegetation coverage quality of $\mathrm{Xi}$ ' an had a trend of degradation in the west of Weiyang District, the east of Xincheng District, the north of Baqiao District and the west of Yanta District; from 2014 to 2017, the quality of vegetation coverage had a trend of improvement in the west of Weiyang District, the north of Baqiao District and the southeast of Yanta District; from 2011 to 2017, the density of vegetation coverage in study area increased and the overall quality improved as well. 


\section{Acknowledgements}

This work was supported by Research on Vegetation Restoration Techniques on Steep Loess Slope in Qianyang, Shaanxi (K303021613).

\section{References}

[1] Li X, Li T, Li H, Qi J, Hu L. Research on the online consumption effect of China's urbanization under population aging background. Sustainability. 2019;11:4349. DOI: 10.3390/su11164349.

[2] Elhoseny H, Elhoseny M, Riad AM, Hassanien AE. A framework for big data analysis in smart cities. Int Conf Adv Machine Learning Technol Appl. 2018;723:405-14. DOI: 10.1007/978-3-319-74690-6_40.

[3] Lv Z, Hu B, Lv H. Infrastructure monitoring and operation for smart cities based on IoT system. IEEE Trans Industrial Informatics. 2019;16:1957-62. DOI: 10.1109/TII.2019.2913535.

[4] Richards DR, Belcher RN. Global changes in urban vegetation cover. Remote Sens. 2020;12(1):23. DOI: $10.3390 /$ rs 12010023 .

[5] Deska I, Mrowiec M, Ociepa E, Michniewski M. Impact of the hydrogel amendment and the dry period duration on the green roof retention capacity. Ecol Chem Eng S. 2020;27:357-71. DOI: 10.2478/eces-2020-0023.

[6] Gdela M, Widomski MK, Musz-Pomorska A. Hydraulic efficency of selected intensive green roof substrates. Ecol Chem Eng A. 2019;26(1-2):37-45. DOI: 10.2428/ecea.2019.26(1-2)4.

[7] Tsai SB. Wang K. Using a novel method to evaluate the performance of human resources in green logistics enterprises. Ecol Chem Eng S. 2019;26(4):629-40. DOI: 10.1515/eces-2019-0045.

[8] Oh RRY, Richards DR, Yee ATK. Community-driven skyrise greenery in a dense tropical city provides biodiversity and ecosystem service benefits. Landscape Urban Planning. 2018;169:115-23. DOI: 10.1016/j.landurbplan.2017.08.014.

[9] Meng XY, Gao X, Li SY, Lei JQ. Spatial and temporal characteristics of vegetation NDVI changes and the driving forces in Mongolia during 1982-2015. Remote Sens. 2020;12:603-28. DOI: 10.3390/rs12040603.

[10] Voorde TVD. Spatially explicit urban green indicators for characterizing vegetation cover and public green space proximity: a case study on Brussels, Belgium. Int J Digital Earth. 2017;10:798-813. DOI:10.1080/17538947.2016.1252434.

[11] Elhoseny M. Multi-object detection and tracking (MODT) machine learning model for real-time video surveillance systems. Circuits Systems Signal Processing. 2020;39:611-30. DOI: 10.1007/s00034-019-01234-7.

[12] Du JQ, Zhao CX, Shu JM, Jiaerheng A, Yuan XJ, Yin JQ, et al. Spatiotemporal changes of vegetation on the Tibetan Plateau and relationship to climatic variables during multiyear periods from 1982-2012. Environ Earth Sci. 2016;75:77. DOI:10.1007/s12665-015-4818-4.

[13] Li ZH, Gao W, Gao ZQ, Shi RH, Liu CH. A study on assessment of urbanization and ecosystem changes based on MODIS time series in Shanghai municipality from 2000 to 2009. Proc SPIE. 2010;7809:78090R. DOI: $10.1117 / 12.858605$.

[14] Liu T, Yang XJ. Monitoring Urban Growth and Land Changes in Beijing, China's Capital City by Remote Sensing: Progress and Challenges: An Interdisciplinary Perspective. In: Challenges Towards Ecological Sustainability in China. 2019:55-67. DOI: 10.1007/978-3-030-03484-9_4.

[15] Hussein SO. Monitoring urban greenness evolution using multitemporal Landsat imagery in the city of Erbil (Iraq). Central European Geol. 2018;62:1-12. DOI: 10.1556/24.61.2018.10.

[16] Lv Z. The security of Internet of drones. Computer Commun. 2019;148:208-14. DOI: 10.1016/j.comcom.2019.09.018

[17] Liang HL, Li WZ, Zhang QP, Zhu W, Chen D, Liu J, et al. Using unmanned aerial vehicle data to assess the three-dimension green quantity of urban green space: A case study in Shanghai, China. Landscape Urban Planning. 2017;164:81-90. DOI: 10.1016/j.landurbplan.2017.04.006.

[18] Hashim H, Abd Latif Z, Adnan NA. Urban vegetation classification with NDVI thresold value method with very high resolution (VHR) PLEIADES Imagery. Int Arch Photogramm. Remote Sens Spat Inf Sci. 2019;237-40. DOI: 10.5194/isprs-archives-XLII-4-W16-237-2019.

[19] Rwanga S, Ndambuki J. Accuracy assessment of land use/land cover classification using remote sensing and GIS. Int J Geosci. 2017;8:611-22. DOI: 10.4236/ijg.2017.84033.

[20] Kussul N, Lavreniuk M, Skakun S, Shelestov A. Deep learning classification of land cover and crop types using remote sensing data. IEEE Geosci Remote Sensing Lett. 2017;14:778-82. DOI: 10.1109/LGRS.2017.2681128. 
[21] Ramasamy B, Yeung MCH. China's one belt one road initiative: The impact of trade facilitation versus physical infrastructure on exports. World Econ. 2019;42:1673-94. DOI: 10.1111/twec.12808.

[22] National Development and Reform Commission of the People's Republic of China. Notice of the National Development and Reform Commission and the Ministry of Housing and Urban-Rural Development on Printing and Distributing the Development Plan of the Guanzhong Plain Urban Agglomeration. 2018. No. 220. Available from: http://www.ndrc.gov.cn/zcfb/zcfbtz/201802/t20180207_876904.html.

[23] John AR. Remote Sensing Digital Image Analysis. 2013. ISBN: 9783642300615. DOI: 10.1007/978-3-642-30062-2.

[24] Xie YC, Sha ZY, Yu M. Remote sensing imagery in vegetation mapping: a review. J Plant Ecol. 2008;1:6240-52. DOI: 10.1093/jpe/rtm005.

[25] Quast R, Albergel C, Calvet JC, Wagner W. A generic first-order radiative transfer modelling approach for the inversion of soil and vegetation parameters from scatterometer observations. Remote Sens. 2019;11:285. DOI: $10.3390 / \mathrm{rs} 11030285$.

[26] Wang J, Du Y, Liu Z, Cheng H. Determining diagnostic indicators for fine-scale short vegetation aboveground biomass inversion using a HVRU-based analysis approach. Ecol Indicators. 2020;111:106033. DOI: 10.1016/j.ecolind.2019.106033.

[27] Zanotta DC, Haertel V, Shimabukuro YE, Renno CD. Linear spectral mixing model for identifying potential missing endmembers in spectral mixture analysis. IEEE Trans Geosci Remote Sens. 2013;52:3005-12. DOI: 10.1109/TGRS.2013.2268539.

[28] Li H, Lei J, Wu J. Evolution analysis of vegetation cover under the disturbance of rare earth mining: a case in Lingbei mining area. J Appl Sci Eng. 2017;20:393-400. DOI: 10.6180/jase.2017.20.3.14.

[29] Adami M, Bernardes S, Arai E, Freitas RM, Shimabukuro YE, Espírito-Santo FDB, et al. Seasonality of vegetation types of South America depicted by moderate resolution imaging spectroradiometer (MODIS) time series. Int J Appl Earth Observation Geoinform. 2018;69:148-63. DOI: 10.1016/j.jag.2018.02.010.

[30] Fassoni-Andrade AC, Zanotta DC, Guasselli LA, Andrade AM. Linear spectral mixing model for estimating optically active components in estuarine waters of Patos Lagoon, Brazil. Int $J$ Remote Sens. 2017;38:4767-81. DOI: 10.1080/01431161.2017.1323281.

[31] Li C, Liu P, Zou C, Sun F, Cioffi JM. Yang L. Spectral-efficient cellular communications with coexistent one-and two-hop transmissions. IEEE Trans Vehicular Technol. 2015;65:6765-72. DOI: 10.1109/TVT.2015.2472456.

[32] Dong Di, Wang Di. Comparisons of ERDAS and ENVI in thematic mapping. 2011 IEEE 3rd Int Conf Communication Software Networks. 2011. DOI: 10.1109/ICCSN.2011.6014623.

[33] Ran P, Eyal BD. Assessing the detection limit of petroleum hydrocarbon in soils using hyperspectral remote-sensing. Remote Sensing Environ. 2019;224:145-53. DOI: 10.1016/j.rse.2019.01.026.

[34] Kumar C, Chatterjee S, Oommen T. Mapping hydrothermal alteration minerals using high-resolution AVIRIS-NG hyperspectral data in the Hutti-Maski gold deposit area, India. Int J Remote Sens. 2020;41:794-812. DOI: 10.1080/01431161.2019.1648906.

[35] Zhao Y, Yang C. Information fusion robust guaranteed cost Kalman estimators with uncertain noise variances and missing measurements. Int $J$ Systems Sci. 2019;50:2853-69. DOI: 10.1080/00207721.2019.1690719.

[36] Bian JH, Li AN, Zhang ZJ, Zhao W, Lei GB, Yin GF, et al. Monitoring fractional green vegetation cover dynamics over a seasonally inundated alpine wetland using dense time series HJ-1A/B constellation images and an adaptive endmember selection LSMM model. Remote Sensing Environ. 2017;197:98-114. DOI: 10.1016/j.rse.2017.05.031.

[37] Zhang J, Liu Y, Zhou DM, Zhang Q, Chen WN. Spatial-temporal character of vegetation cover and its influence factors in the Shule River Basin China, during 1985-2011. Human Ecol Risk Assess: Int J. 2020;26:608-20. DOI: 10.1080/10807039.2018.1528437.

[38] Peng J, Liu YH, Shen H, Han YN, Pan YJ. Vegetation coverage change and associated driving forces in mountain areas of Northwestern Yunnan, China using RS and GIS. Environ Monit Assess. 2012;184:4787-98. DOI: 10.1007/s10661-011-2302-5.

[39] Liu X, Zhou W, Bai Z. Vegetation coverage change and stability in large open-pit coal mine dumps in China during 1990-2015. Ecol Eng. 2016;95:447-51. DOI: 10.1016/J.ECOLENG.2016.06.051.

[40] Zhao K, Jin BX, Fan H, Song WW, Zhou SY, Jiang YY. High-performance overlay analysis of massive geographic polygons that considers shape complexity in a cloud environment. Int $\mathrm{J}$ Geo-Information. 2019;8:290. DOI: 10.3390/ijgi8070290.

[41] Yan LB, He RX, Kašanin-Grubin M, Luo G, Peng H, Qiu JX. The dynamic change of vegetation cover and associated driving forces in Nanxiong Basin, China. Sustainability. 2017;9:443. DOI: 10.3390/su9030443. 
[42] Liu SY, Huang SZ, Xie YY, Wang H, Huang Q, Leng GY, et al. Spatial-temporal changes in vegetation cover in a typical semi-humid and semi-arid region in China: Changing patterns, causes and implications. Ecol Indicators. 2019;98:462-75. DOI: 10.1016/j.ecolind.2018.11.037.

[43] Du JQ, Fu Q, Fang SF, Wu JH, He P, Quan ZJ. Effects of rapid urbanization on vegetation cover in the metropolises of China over the last four decades. Ecol Indicators. 2019;107:105458. DOI: 10.1016/j.ecolind.2019.105458.

[44] Yang Z, Song J, Cheng D, Xia J, Li Q, Ahamad MI. Comprehensive evaluation and scenario simulation for the water resources carrying capacity in Xi'an city, China. J Environ Manage. 2019;230:221-33. DOI: 10.1016/j.jenvman.2018.09.085. 